\title{
Impact of Capacitor Placement on Medium Voltage for Improve the Power Factor
}

\author{
Nattachote Rugthaicharoencheep*, Chatpong Boobpa \\ Rajamangala University of Technology Phra Nakhon, Bangkok, 10800 Thailand. \\ * Corresponding author. Tel.: +66 613536426; email: nattachote.r@rmutp.ac.th \\ Manuscript submitted June 22, 2018; accepted October 21, 2018. \\ doi: 10.17706/ijcee.2019.11.1.20-26
}

\begin{abstract}
This paper presents impact of capacitor placement on medium voltage for improve the power factor value to $\mathrm{AC}$ motor. In present, the electrical power system has focus on improving the Power Factor significantly because it is the major key to increase or decrease the applicable expenses. The power system that has low power factor will have a lot of loss to system, especially when electrical voltage is on voltage medium. When power factor has been adjusted, the system can take more loads and electrical power loss is decreased. It also decreases voltage in power line and decreases electrical bills at the same time. This article presents the theory to analyze data from motor that needs to adjust a power factor so that it pass electricity authority standard by considering the efficiency of the motor so that the consumers do not have to pay the penalty of the power factor value differences to the Electricity Authority. Furthermore, it can help decrease the bills of unstandardized electrical power which occurred in the system and enable the system to take more loads at the same time.
\end{abstract}

Key words: Capacitor placement, medium voltage, power system, power factor.

\section{Introduction}

The system has a low power factor will result in the ability to power down the transformer. Because of the power transformer to be offset by the power loss that occurs within the system [1]. If the ability to supply power to the load of the transformer is almost full. You will not be able to power the electrical load has increased [2] Although power the address will not be filled. Moreover, in cases where the electric wires are very long. It will cause electric currents in power lines has increased. And heat in a line up. Result in the power loss. The size of the current squared. And, most importantly, power users will want to add to the cost of electricity.

A fault occurred in the electrical system is causing the change in voltage quicker. The voltage of the electrical system was distorted from the original. The fault of the power system can be classified into several types based on birth. Timing and magnitude of changes in voltage or current. Such as a power outage over voltage drop over current in power systems to improve the power factor is particularly important. As a result, the costs increase or decrease. Power system with low power factor will be lost in the system. Therefore, the equipment used needs to be larger. Making the cost of capital equipment increased. Therefore, to improve the power factor is high, so there is no need for office buildings and industrial plants. Want real power and reactive power. In order to work [3].

From the above reasons, the need for improved power factor, voltage stability of the power system when it 
is supplied to the load. Thus prepared, therefore, to design a device to compensate reactive power to the power factor control [4]. To reduce the voltage in the power system allows the system to load the real power even more.

\section{Power Factor}

In power systems to improve the power factor is important [5]. The cost is increased or decreased. Power system with low power factor. Power factor correction with low power losses. The devices require larger. To improve the power factor to a value higher. Are essential to power system. This power is consistent with the real power and reactive power. Improving power factor, Is to improve the power factor to values approaching 1.0. Is to reduce the size of the active power, reactive power appears to have coincided with the real power [6]. In practice, most of the load will feature a mix of resistance inductor. Is made current lags voltage [7]. The Power Series Active in common, it is often a source of reactive power inductors. When considered as a whole, you will find that the power of the reactive capacitance. The overall system power factor is low. To improve power factor by the capacitor in parallel with the load or source. So the reactive power of the power of tough against capacitor [8]-[10] As a result, apparent power decreases. This principle is to improve the power factor by adjusting the reactive power. For the apparent power a similar bundle into real power. Power factor value that is equal to 1.0. The best of power quality. Reduce the power loss is shown in Fig. 1.
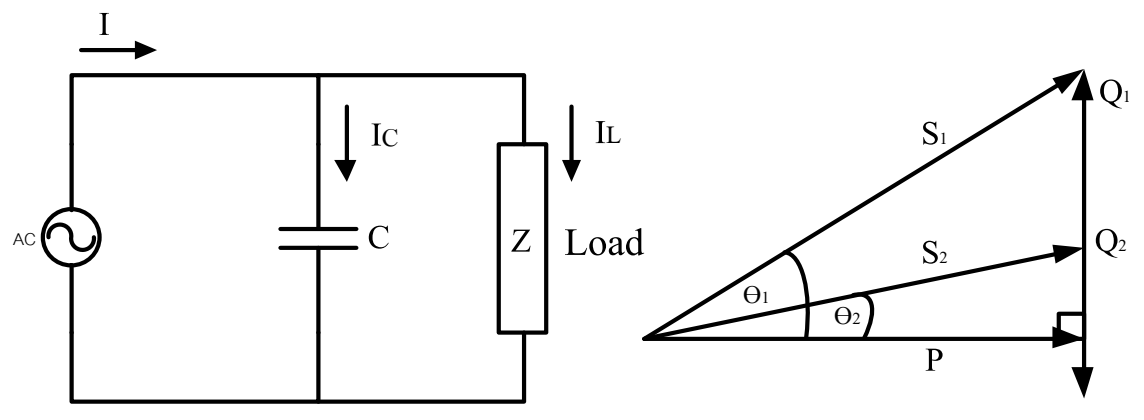

Fig. 1. Connect a capacitor in order to improve the power factor value.

Improvement of power factor compensation. From $P F_{1}$ to $P F_{2}$ can calculate the coordinates of the capacitor unit in reactive power from the equation as follows.

$$
\begin{gathered}
P F_{1}=\frac{k \mathrm{~W}}{k \mathrm{VA}_{1}}=\cos \theta_{1}, P F_{2}=\frac{k \mathrm{~W}}{k \mathrm{VA}_{2}}=\cos \theta_{2} \\
\tan \theta_{1}=\frac{Q_{1}}{P}, \tan \theta_{2}=\frac{Q_{2}}{P} \\
Q_{1}=P \tan \theta_{1}=k \mathrm{~W} \times \tan \theta_{1} \\
Q_{2}=P \tan \theta_{2}=k \mathrm{~W} \times \tan \theta_{2}
\end{gathered}
$$

Therefore, the size of the capacitors from 


$$
\begin{aligned}
(k \mathrm{Var})=Q_{1}-Q_{2} & =k \mathrm{~W} \tan \theta_{1}-k \mathrm{~W} \tan \theta_{2} ? \\
& =k \mathrm{~W}\left(\tan \theta_{1}-\tan \theta_{2}\right)
\end{aligned}
$$

Using the Table 1 , which is the value in the table $\tan \theta_{1}-\tan \theta_{2}$ for calculating the size of the capacitor? (kVar) As if want to improve the power factor from $P F_{1}=0.85$ up to $P F_{2}=0.95$ Results $\tan \theta_{1}-\tan \theta_{2}=0.29$.

\begin{tabular}{|c|c|c|c|c|c|c|c|c|c|c|c|c|c|c|}
\hline \multirow{15}{*}{$\tan \theta_{1}$} & & & & & & & & $\tan \theta_{2}$ & & & & & & \\
\hline & & 0.8 & 0.85 & 0.90 & 0.91 & 0.92 & 0.93 & 0.94 & 0.95 & 0.96 & 0.97 & 0.98 & 0.99 & 1.00 \\
\hline & 0.80 & 0 & 0.13 & 0.27 & 0.29 & 0.32 & 0.36 & 0.39 & 0.42 & 0.46 & 0.50 & 0.55 & 0.61 & 0.75 \\
\hline & 0.85 & & 0 & 0.14 & 0.16 & 0.19 & 0.23 & 0.26 & 0.29 & 033 & 0.37 & 0.42 & 0.48 & 0.62 \\
\hline & 0.90 & & & 0 & 0.03 & 0.06 & 0.09 & 0.12 & 0.16 & 0.19 & 0.23 & 0.28 & 0.34 & 0.48 \\
\hline & 0.91 & & & & 0 & 0.03 & 0.06 & 0.09 & 0.13 & 0.16 & 0.21 & 0.25 & 0.31 & 0.46 \\
\hline & 0.92 & & & & & 0 & 0.03 & 0.06 & 0.10 & 0.13 & 0.18 & 0.22 & 0.28 & 0.43 \\
\hline & 0.93 & & & & & & 0 & 0.03 & 0.07 & 0.10 & 0.15 & 0.19 & 0.25 & 0.40 \\
\hline & 0.94 & & & & & & & 0 & 0.03 & 0.0 & 0.11 & 0.16 & 0.22 & 0.36 \\
\hline & 0.95 & & & & & & & & 0 & 0.04 & 0.08 & 0.13 & 0.19 & 0.33 \\
\hline & 0.96 & & & & & & & & & 0 & 0.04 & 0.89 & 0.15 & 0.29 \\
\hline & 0.97 & & & & & & & & & & 0 & 0.05 & 0.11 & 0.25 \\
\hline & 0.98 & & & & & & & & & & & 0 & 0.06 & 0.20 \\
\hline & 0.99 & & & & & & & & & & & & 0 & 0.14 \\
\hline & 1.00 & & & & & & & & & & & & & 0 \\
\hline
\end{tabular}

Table 1. $\tan \theta_{1}-\tan \theta_{2}$ for Calculating the Size of the Capacitor

\section{Design of Reactive Power Compensation}

Design and assembly of reactive power compensation device to control factor. Consider the strength and the safety of the user. The layout of the design is shown in Fig. 2.

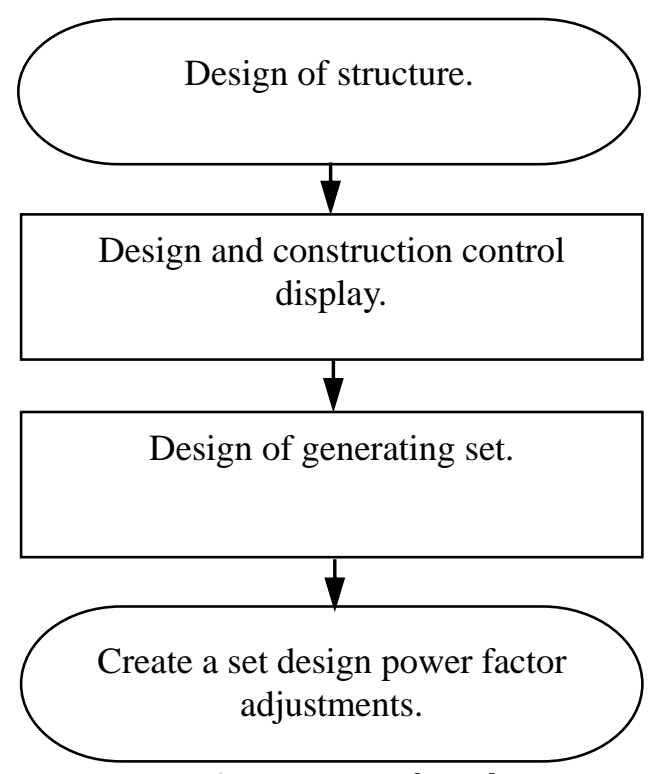

Fig. 2. Diagram of work. 


\section{Equipment}

Capacitor compensate the reactive power in the circuit it uses a capacitor size $0.83 \mathrm{kVAR}$ the 3 in electrical 1 phase and 2.5 kVAR the 2 and 5 kVAR the 1 in a 3-phase electrical system [11].

Magnetically's contactor is used to cut the capacitors to improve power factor.

Fuse size 4 A surge protector helps in making electrical equipment damaged

Current Transformer size 30 / 5 A used in measuring current in the circuit to control factor is know in RG3-12C control factors are accurately.

Circuit breaker $30 \mathrm{~A}$ size used in editing main power into the circuit power factor compensation.

Selector switch used to compensate power factor up both automatic and controlled by the individual.

Pilot Lamp used to display the status of the capacitor compensation value is in electrical assembly

Power factor controller model RG3-12C used in processing and improve the power factor of a power up automatically. The input parameters to control power factor can precisely.

The compensation capacitor if the capacitor that is too high may result in a power factor of 1.0 and may outweigh the potential damage to equipment in the power system. The revised power factor capacitors are required on many measures calculated to select as appropriate. By installing capacitors in series to improve the power factor is used. And a circuit diagram showing the overall improvement of the power factor in this article are shown in Fig. 3.

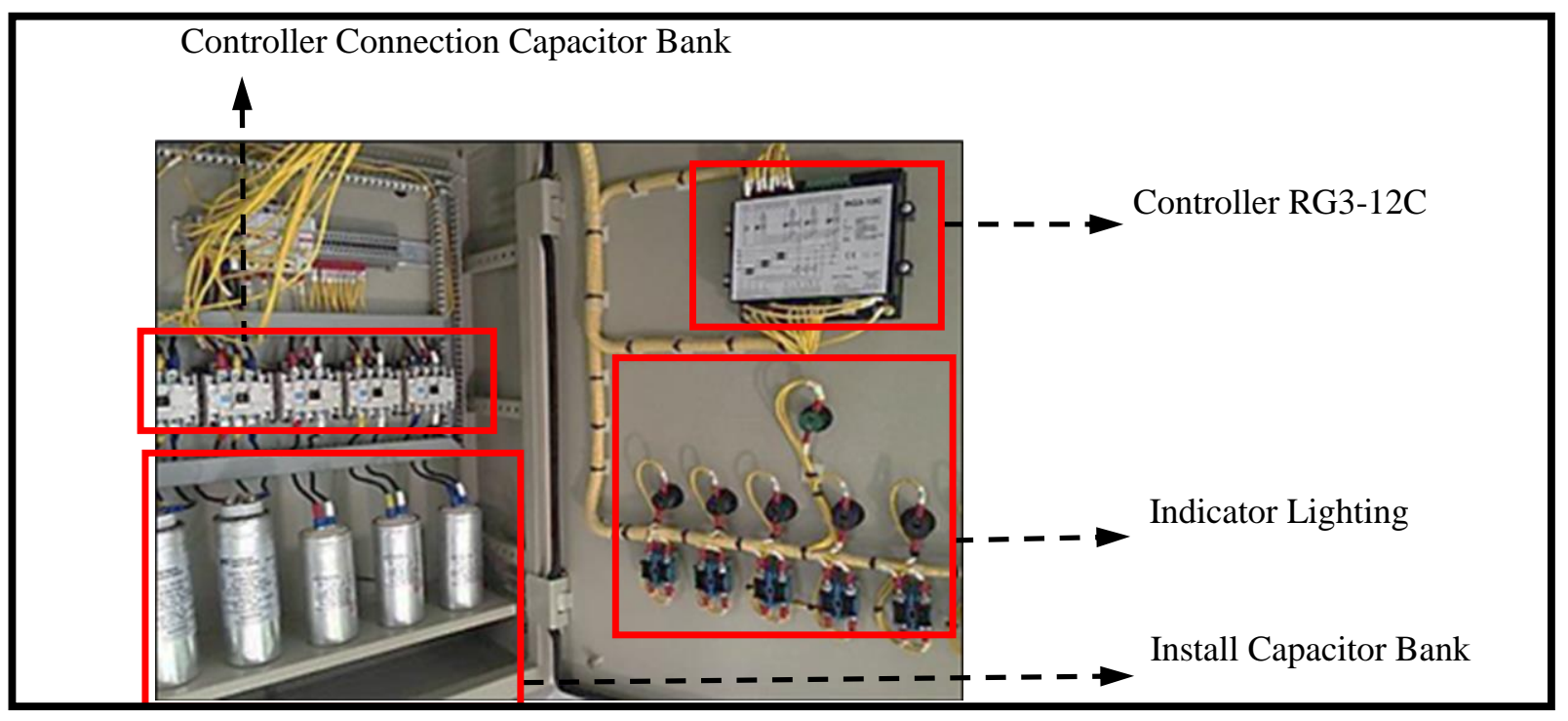

Fig. 3. Control components and power series.

\section{Case Study}

Connect the three phase AC power supply with Equipment to compensate the reactive power. [10] To control the power factor. [9] Examine the accuracy of the circuiting input the parameter to the power factor control RG3-12C. Then connect it to the test load. In this improvement, [4] the central capacitor installation was implemented as it was automatic easy to monitor and record the result in the table. Bring the value to the conclusion of the research is shown in Fig. 4.

The results in Table 2 and Table 3. The results are in accordance with the purpose of designing a device to compensate reactive power source. Seen that before power factor correction capacitors in parallel to the system to pay for treatment active is lower than 0.85 , including parameters were higher. Such as electricity and real power. As a result, the power loss in the system is high as well. But after the parallel capacitor Log in to power compensation active. 


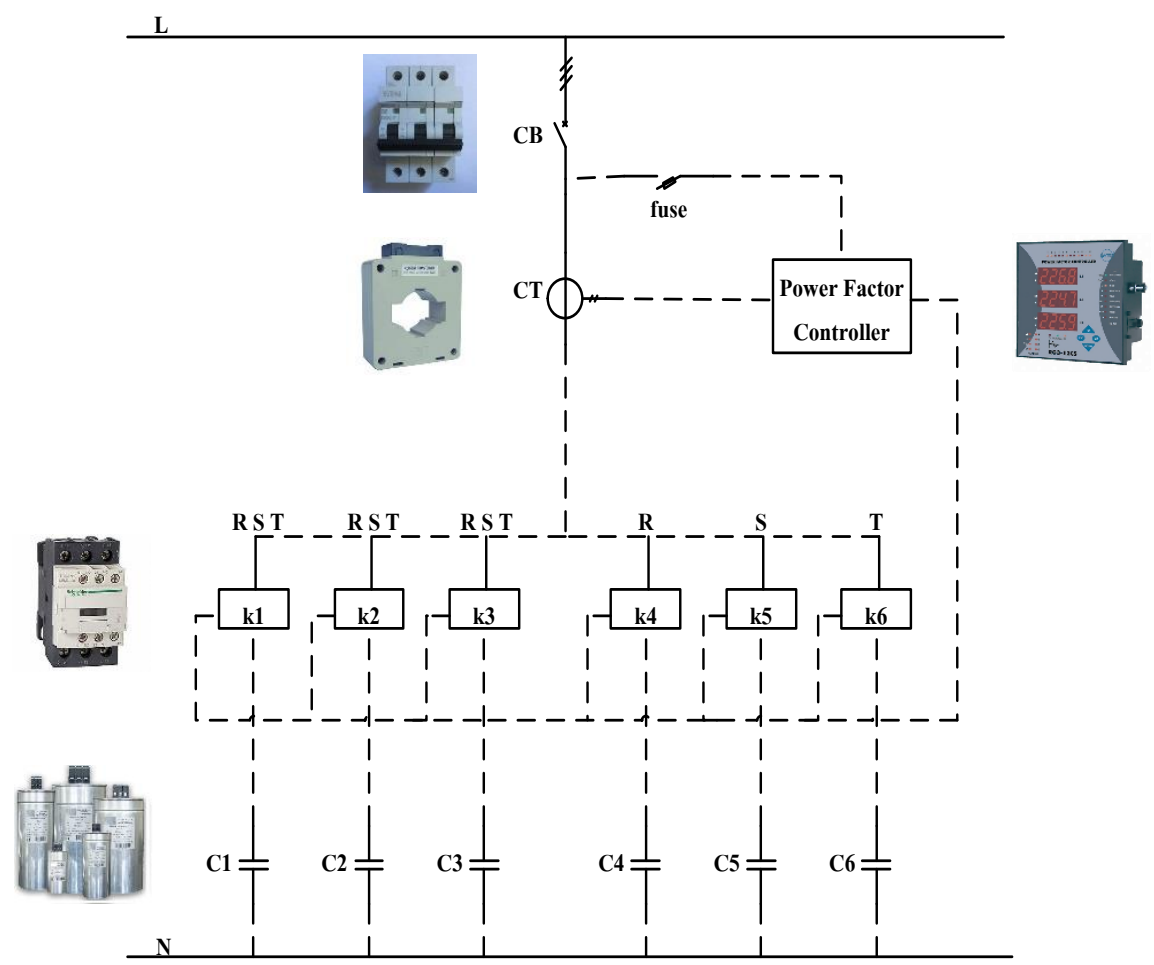

Fig. 4. Circuit of series improve overall factor.

Table 2. Value from the Meter before / after the Equipment into the Motor 1 Phase

\begin{tabular}{|c|c|c|c|c|c|c|c|c|}
\hline & \multicolumn{2}{|c|}{ L1 } & \multicolumn{2}{|c|}{ L2 } & \multicolumn{2}{|c|}{ L3 } & \multicolumn{2}{|c|}{ total } \\
\hline & before & after & before & after & before & after & before & after \\
\hline V & 233.4 & 231.1 & - & - & - & - & 233.4 & 231.1 \\
\hline I & 14.04 & 12.33 & - & - & - & - & 14.04 & 12.33 \\
\hline W & 2592 & 2548 & - & - & - & - & 2592 & 2548 \\
\hline VAR & 1840 & 1126 & - & - & - & - & 1840 & 1126 \\
\hline $\cos \theta$ & 0.50 & 0.929 & - & - & - & - & 0.50 & 0.929 \\
\hline VA & 3241 & 2863 & - & - & - & - & 3241 & 2863 \\
\hline
\end{tabular}

Table 3. Value from the Meter before / after the Equipment into the Motor 3 Phase

\begin{tabular}{cccccccccc}
\hline \multicolumn{10}{c}{ 31-phase motors } \\
\hline & \multicolumn{8}{c}{ L2 } & \multicolumn{2}{c}{ L3 } & \multicolumn{2}{c}{ total } \\
& before & after & before & after & before & after & before & after \\
\hline V & 237.4 & 237.3 & 235.7 & 235.8 & 234.9 & 234.2 & 236 & 235.8 \\
I & 2.772 & 0.564 & 2.760 & 0.582 & 1.548 & 1.680 & 2.360 & 0.942 \\
W & 184.2 & 63.0 & 187.2 & 33.6 & 142.2 & 42.0 & 514.2 & 96.6 \\
VAR & 627.6 & 94.8 & 621.0 & 106.8 & 337.2 & 388.8 & 1591 & 592 \\
COSE & 0.294 & 0.537 & 0.297 & 0.290 & 0.378 & 0.108 & 0.308 & 1.0 \\
VA & 625.8 & 132.6 & 646.2 & 133.2 & 363.6 & 394.8 & 1665 & 661.2 \\
\hline
\end{tabular}

\section{Conclusion}

This research paper presented to compensate for reactive power control of factor with parallel capacitor log control. From the comparison between experimental results before compensation reactive power The factor is in the system is lower than the standard limit is lower than 0.85 but when the compensation value of reactive power control used for the show that is value is based on a standard is higher 0.85 or above. 


\section{Acknowledgment}

The authors are very grateful acknowledgement the Rajamangala University of Technology Phra Nakhon (Bangkok, Thailand) for the sponsorship provided to this research.

\section{References}

[1] Celtekligil, U. (2008). Capacitive power factor and power quality correction of a light rail transportation system. Proceedings of the 50th International Symposium ELMAR 2008: Vol 2. (pp. 415-418).

[2] Tou, M., Al-Haddad, K., Olivier, G., \& Rajagopalan, V. (1994). A new three-phase unity power factor boost rectifier with capacitive-type input. Proceedings of the IEEE 4th Workshop on Computer in Power Electronics (pp. 256-265).

[3] Leung, A. K. N., Mok, P. K. T., Ki, W. H., \& Sin, J. K. O. (1999). Damping-factor-control frequency compensation technique for low-voltage low-power large capacitive load applications. Proceedings of the ISSCC 1999 (pp.158-159).

[4] Barbi, I., Fagundes, J. C., \& Cruz, C. M. T. (1994). A low cost high power factor three-phase diode rectifier with capacitive load. Proceedings of the APEC-94 (pp. 745-751).

[5] Zackrison, H. B. (1989). Reducing energy consumption frees up electrical system capacity. Proceedings of the IECEC-89: Vol 4. (pp. 1755-1765).

[6] Su, C. L., Lin, M. C., \& Liao, C. H. (2012). A energy-savings evaluation method to justify automatic power factor compensators on marine vessels. Proceedings of the 2012 IEEE Industry Applications Society Annual Meeting (IAS) (pp. 1-10).

[7] Deaconu, S. I., Popa, G. N., \& Babau, R. (2014). Study, design and industrial implementation of capacitive power factor controller for large load fluctuations in steel industry. Proceedings of the 2014 International Conference and Exposition on Electrical and Power Engineering (EPE) (pp. 962-967).

[8] Shateri, H., Ghorbani, M., \& Mohammad-Khani, A. H. (2011). Load flow method for distribution networks with automatic power factor controller. Proceedings of the IEEE International Universities' Power Engineering Conference (UPEC) (pp. 1-6).

[9] Suwat, K., Mongkol, K., \& Anawach, S. (2012). A digital implementation of novel single phase AC-AC converter with power factor control. Proceedings of the IEEE International Conference on Electrical Machines and Systems (ICEMS) (pp. 1-6).

[10] Davidson, C. D. (2015). Single stage true bridgeless AC/DC power factor corrected converter. Proceedings of the IEEE International Telecommunications Energy Conference (INTELEC) (pp. 1-6).

[11] Pires, V. F., Costa, D., Marques, G., \& Silva, J. F. (2008). An anti-resonance active method for a power factor correction capacitive system. Proceedings of the IEEE Region 8th International Conference on SIBIRCON 2008 (pp. 133-138).

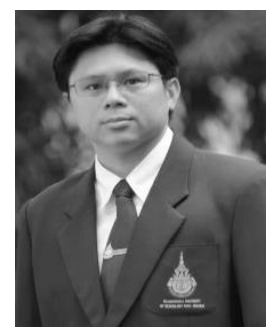

Nattachote Rugthaicharoemncheep is an associate professor in the Department of Electrical Engineering at the Rajamangala University of Technology Phra Nakhon (RMUTP), Bangkok, Thailand. He obtained his Ph.D in electrical engineering from King Mongkut's Institute of Technology North Bangkok, Thailand in October 2010. His current research areas are power system planning and operation, optimization techniques, distributed generation (DG), as well as the development of distribution system with DGs. His contributions to the subject include many books, journals, and conferences. Currently, He is also a senior member of IEEE. 


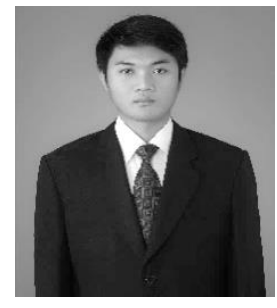

Chatpong Boobpa is a master student in electrical engineering program at Department of Electrical Engineering at the Rajamangala University of Technology Phra Nakhon (RMUTP), Bangkok, Thailand. He obtained his B.Eng in Rajamangala University of Technology Phra Nakhon, Thailand in May 2015. His current research areas are power system planning and operation. Currently, $\mathrm{He}$ is an electrical engineer at the Auto-Info Company Thailand. 